The uniqueness of finite geometries with less than 6 points on every line was first proved by J. H. M. Wedderburn and O. Veblen [4]. The uniqueness of finite geometries with 6 points on every line was first demonstrated by C. R. MacInnes [5] in a rather laborious tactical enumeration of cases.

\title{
REFERENCES
}

1. G. Tarry, Le problème de 36 officiers, Compte Rendu de l'Association Francaise pour l'Advancement de Science Naturel vol. 1 (1900) pp. 122-123, vol. 2 (1901) pp. 170-203.

2. R. C. Bose, On the application of the properties of Galois fields to the problem of construction of hyper-Graeco-Latin squares. Sankhya, Indian Journal of Statistics, vol. 3 (1938) pp. 323-338.

3. H. B. Mann, The construction of sets of orthogonal Latin squares, Annals of Mathematical Statistics vol. 13 (1942) pp. 418-423.

4. J. H. M. Wedderburn and O. Veblen, Non-Desarguesian and non-Pascalian geometries, Trans. Amer. Math. Soc. vol. 8 (1907) pp. 379-388.

5. C. R. MacInnes, Finite planes with less than eight points on a line, Amer. Math. Monthly vol. 14 (1907) pp. 171-174.

BARD COLLEgE

\section{SOME THEOREMS ON CO-TERMINAL ARCS}

\section{R. H. SORGENFREY}

It is the purpose of this note to prove certain properties of sums of simple arcs which have one or both end points in common. The investigation was undertaken to answer a question, that of the validity of Theorem 3 below, raised by Miss Harlan C. Miller. An example is included to show that two of the results obtained are not valid for irreducible continua in general.

TheOREM 1. If $H$ and $K$ are two distinct arcs from $A$ to $B$, then each point of $H+K-H \cdot K$ belongs to a simple closed curve lying in the closure of $H+K-H \cdot K$.

PRoof. Let $P$ be any point of $H+K-H \cdot K=N$, and let $S$ be the component of $N$ which contains it. The set $S$ is an arc segment; let its end points be $X$ and $Y$. Suppose that no simple closed curve lying in $\bar{N}$ contains $P$. Then $\bar{N}-S$ contains no continuum containing both $X$ and $Y$, for if it did it would contain an arc from $X$ to $Y$, and this arc plus $S$ would be a simple closed curve lying in $\bar{N}$ and contain-

Presented to the Society, December 31, 1941; received by the editors October 29. 1943. 
ing $P$. There exist, therefore, two disjoint closed sets $U$, containing $X$, and $V$, containing $Y$, such that $\bar{N}-S=U+V$. The components of $H+K-(U+V)$ are, except for $S$, subsets of $H \cdot K$ and hence are segments of arcs or segments plus one end point. Let $U^{\prime}$ be $U$ plus all components of $H+K-(U+V)$ which have no end points in $V$, and let $V^{\prime}$ be $V$ plus all components of $H+K-(U+V)$ which have no end points in $U$. Then $U^{\prime}$ and $V^{\prime}$ are closed and disjoint. Hence the components of $H+K-\left(U^{\prime}+V^{\prime}\right)$ are finite in number since each of them has one end point in $U^{\prime}$ and the other in $V^{\prime}$. Since all these segments but $S$ are subsets of both $H$ and $K$, while $S$ is a subset of only one of these, it follows that the number of segments between $U^{\prime}$ and $V^{\prime}$ of one of the $\operatorname{arcs} H$ and $K$ is even, while that of the other is odd. This is impossible since both $H$ and $K$ have $A$ and $B$ as end points.

THEOREM 2. If $H, K$, and $L$ are three distinct arcs from $A$ to $B$, then there are two of them neither of which is a subset of the sum of the other two.

Proof. Suppose that $H$ is a subset of $K+L$ and $K$ is a subset of $H+L$; then $H+K-H \cdot K=N$ is a subset of $L$. This implies that $\bar{N}$ is a subset of $L$. By Theorem 1 there is a simple closed curve in $\bar{N}$. Hence the arc $L$ contains a simple closed curve. It follows from this contradiction that one of the arcs $H$ and $K$ fails to be a subset of the sum of the other two. Suppose $H$ does. Then it may be shown in the same way that one of the $\operatorname{arcs} K$ and $L$ fails to be a subset of the sum of the other two, and the theorem is proven.

THEOREM 3. If $H, K$, and $L$ are three arcs emanating from a point $A$ and no one of them is a subset of any other, then there is one of them which fails to be a subset of the sum of the other two.

PRoof. Suppose that each of the $\operatorname{arcs} H, K$, and $L$ is a subset of the sum of the other two. Let the end points, other than $A$, of $H, K$, and $L$ be $B_{H}, B_{K}$, and $B_{L}$, respectively. No two of the points $B_{H}, B_{K}$, and $B_{L}$ coincide, for if they did a contradiction could be reached, as it was in the proof of Theorem 2, by the use of Theorem 1. If $B_{H}$ did not belong to $L$, some neighborhood with respect to $H+K+L$ of $B_{H}$ would be a subset of $H \cdot K$, which is impossible unless $B_{H}$ and $B_{K}$ coincide. Hence $B_{H}$ and, similarly, $B_{K}$ belong to $L$. Suppose that $B_{H}$ precedes $B_{K}$ in the order $A B_{L}$ on $L$. Not every point of the interval $A B_{H}$ of $L$ belongs to $H$, or if it did $H$ would be a subset of $L$. Hence there exists a segment $S$ of the interval $A B_{L}$ of $L$ which contains no point of $H$. Let $X$ and $Y$ be the end points of $S$. Denote the intervals of $A X$ and $Y B_{L}$ of $L$ by $U$ and $V$, respectively. A contradiction 
may now be reached by an argument very similar to the last part of the proof of Theorem 1 .

That Theorems 2 and 3 do not remain true if the word "arcs" in their hypotheses is replaced by the words "irreducible continua" is shown by the following example.

Example. There exist in the plane two points $A$ and $B$ and three continua, each irreducible from $A$ to $B$, such that each of them is a subset of the sum of the other two.

Construction. Let $C$ be a unit circle and $S$ be $C$ plus its interior. Denote by $I_{H}, I_{K}$, and $I_{L}$ three mutually exclusive and non-abutting segments of $C$. For each positive integer $n$ let $D_{H n}, D_{K n}$, and $D_{L_{n}}$ be simple domains lying in $S-C$ such that if $X$ is $H, K$, or $L$ and $E_{X_{n}}=D_{X_{1}}+D_{X_{2}}+\cdots+D_{X_{n}}$, then (1) $\bar{D}_{X_{1}} \cdot C=\bar{I}_{X}$, (2) $\bar{D}_{X_{n}} \cdot C=0$ if $n>1$, (3) $\bar{D}_{X_{n}} \cdot \bar{D}_{X(n+1)}$ is an arc of length less than $1 / n$, (4) $\bar{D}_{X_{n}} \cdot \bar{D}_{X_{(n+i)}}$ $=0$ if $i>1$, and (5) each point of $S$ is within $1 / n$ units of $E_{X_{n}}$. Furthermore let $D_{X_{n}}$ be such that $E_{H n} \cdot E_{K_{n}}=E_{K_{n}} \cdot E_{L_{n}}=E_{L_{n}} \cdot E_{H n}$. Then if $M_{X}=S-\left(I_{X}+D_{X 1}+D_{X 2}+\cdots\right)$, it follows that each of the sets $M_{H}$, $M_{K}$, and $M_{L}$ is an indecomposable continuum, ${ }^{1}$ that each of them is a subset of the sum of the other two, and that no one of them is a subset of any other. It is readily seen that there are two points between which each of these continua is irreducible.

University of California at Los Angeles

1 It will be noted that the construction of the separate continua $H, K$, and $L$ was made by the "canal" method. See K. Yoneyama, Theory of continuous set of points, Tôhoku Math. J. vol. 12 (1917) pp. 60-62. 